\title{
Mecanismos neuro-hormonais envolvidos na regulação do apetite ao sódio: alguns aspectos
}

\author{
Neuro-hormonal mechanisms involved within sodium appetite: some aspects
}

Silmara Formenti', Eduardo Colombari',2

\begin{abstract}
Resumo
0 sódio é o principal ín do líquido extracelular e tem primordial importância para diversas funções fisiológicas. Manter a concentração plasmática do sódio dentro dos limites fisiológicos é vital para diversas espécies de animais, inclusive os humanos. Portanto, é fundamental que hajam mecanismos responsáveis pela monitorização e manutenção de níveis adequados de sódio no plasma. 0 apetite ao sódio, o comportamento que comanda a ingestão de sal, é estimulado por situações de deficiência sistêmica de sódio. Ao longo de décadas, diversos estudos foram desenvolvidos a fim de compreender os mecanismos neurais e hormonais envolvidos no controle desse comportamento. No entanto, muitas questões relacionadas a esse assunto ainda permanecem desconhecidas e a cada dia surgem novas evidências. Neste trabalho, foram revistos os fatos históricos, mecanismos neurais, hormonais e os achados mais recentes envolvendo apetite ao sódio.
\end{abstract}

Palavras-chave: sódio; sódio na dieta; hiponatremia; angiotensina II; aldosterona.

\begin{abstract}
Sodium is the most important ion of the extracellular fluid and plays an important role for many physiological functions. Maintain the sodium concentration in the plasma around of its physiological values is vital for many species of animals, including humans. Therefore, it is important that have responsible mechanisms for monitorization and maintenance of appropriated levels of plasmatic sodium. Sodium appetite, the behavioral drive to ingest salt, is stimulated by systemic sodium deficiency. Over the past decades, several studies have been performed in order to understand the neural and hormonal mechanisms involved in the control of this behavior. However, many questions related to this issue are still unknown and everyday new evidences arise. Here, we review historical facts, neural and hormonal mechanisms and recent findings involving sodium appetite
\end{abstract}

Keywords: sodium; sodium, dietary; hyponatremia; angiotensin II; aldosterone.

Recebido em: 03/03/2011

Revisado em: 29/07/2011

Aprovado em: 02/08/2011

\footnotetext{
Trabalho realizado na Universidade Federal de São Paulo - Escola Paulista de Medicina (UNIFESP-EPM) - São Paulo (SP), Brasil

Departamento de Fisiologia da Universidade Federal de São Paulo - Escola Paulista de Medicina (UNIFESP-EPM) - São Paulo (SP), Brasil.

2 Disciplina de Fisiologia da Faculdade de Medicina do ABC (FMABC) - Santo André (SP), Brasil.

Endereço para correspondência: Eduardo Colombari - Avenida Lauro Gomes, 2000 - Sacadura Cabral - CEP: 09060-870 - Santo André (SP), Brasil -

E-mail: eduardo.colombari@pesquisador.cnpq.br.

Fonte de financiamento: nenhuma.

Conflito de interesse: nada a declarar.
} 


\section{Introdução}

O apetite ao sódio é comportamento motivado, inato e está presente em diversas espécies de animais ${ }^{1,2}$, inclusive em humanos ${ }^{3}$. Como o próprio nome indica, é um comportamento que estimula os animais a buscarem e ingerirem alimentos ou soluções que contenham sódio, que normalmente ocorre em resposta à deficiência de sódio no organismo.

Os estudos pioneiros de Curt Richter (1936) foram o marco inicial para os estudos envolvendo o apetite ao sódio. Richter observou que ratos adrenalectomizados (ADX) morriam rapidamente quando mantidos em dieta hipossódica, e que quando permitido livre acesso a salina hipertônica esses animais apresentavam robusta ingestão de sódio e isso os mantinha vivos ${ }^{1}$. Richter concluiu então que o apetite ao sódio apresentado pelos animais ADX era fruto da deficiência do íon sódio e que esse comportamento poderia indicar a presença de distúrbios endócrinos, pois humanos em situações que sabidamente causam alterações hormonais, como a doença de Addison e a gravidez, apresentavam aumento na preferência por alimentos salgados ${ }^{1}$.

Descobertas adicionais vieram com estudos subsequentes nos quais se observou que o apetite por cloreto de sódio $(\mathrm{NaCl})$ desenvolvido por ratos ADX era específico para soluções contendo sódio, e que esse comportamento era abolido depois que estes animais recebiam implantes de tecido adrenal ou depois da administração exógena de acetato de desoxicorticosterona (DOCA $)^{4}$. A partir de então, Richter pôde concluir que as glândulas adrenais e os hormônios por ela produzidos estavam diretamente relacionados ao controle da ingestão de sódio.

Ademais, Rice e Richter ${ }^{5}$ observaram que ao contrário do que ocorria em animais $\mathrm{ADX}$, animais intactos desenvolviam apetite ao sódio quando tratados com DOCA. Todos esses resultados mostraram a importância dos hormônios produzidos pelas glândulas adrenais no controle da ingestão de sódio e deram início a inúmeras pesquisas que buscaram investigar os mecanismos envolvidos no apetite ao sódio induzido por mineralocorticoides e outros fatores que também poderiam contribuir para esse comportamento. O sódio é o principal íon do líquido extracelular (LEC) e tem função fundamental na manutenção da osmolaridade e do volume sanguíneo, e, consequentemente, da pressão arterial. Perturbações na homeostase do sódio podem ter consequências graves, podendo inclusive levar a morte como demonstrado inicialmente por Richter ${ }^{1}$. Além do estudo em ratos $\mathrm{ADX}^{1}$, outros estudos demonstraram a importância do sódio. No trabalho de Orent-Keiles ${ }^{6}$, ratos criados em dieta hipossódica apresentaram rápida perda de peso, problemas oculares, atraso na maturidade sexual em fêmeas e morte de 100\% dos animais entre 18 e 21 semanas após o início do protocolo de privação de sódio. A análise necroscópica revelou diversos problemas, tais como: perda de tecido adiposo, atrofia muscular, infecção pulmonar, alterações no fígado, nas glândulas adrenais e nos rins, ossos rarefeitos, redução das cartilagens, redução no tamanho dos testículos, entre outras ${ }^{6}$.
Em humanos, o apetite ao sódio é assunto bem menos estudado do que em animais, talvez pela dieta ser normalmente rica em sódio ou por ser uma sensação difícil de ser reconhecida. Porém, há relatos na literatura que comprovam a presença de apetite ao sódio em humanos e mostram os distúrbios que ocorrem devido à deficiência orgânica desse íon. O relato mais conhecido na literatura foi descrito em 1940 por Wilkins e Richter ${ }^{7}$. Esse caso relata a história de uma criança de três anos e meio de idade, hospitalizada com quadro de puberdade precoce e que, após sete dias de internação, houve morte súbita. Na autópsia foi descoberto que a criança sofria de insuficiência adrenal. Seus pais relataram a Wilkins e Richter que a criança apresentava insaciável vontade de ingerir sal, sendo que uma das primeiras palavras que aprendeu foi "sal". Esse comportamento foi o que a manteve viva até então, pois, durante a internação, a dieta hipossódica oferecida no hospital foi insuficiente para compensar a perda renal de sódio resultante da ausência de produção de mineralocorticoides pelas glândulas adrenais e com isso a criança rapidamente morreu? ${ }^{7}$.

Esses estudos mostram as consequências decorrentes da deficiência de sódio em humanos e animais, salientando a importância desse íon na manutenção da homeostase do organismo. Além disso, fica claro a importância da existência de um sistema de detecção dos níveis orgânicos de sódio que desencadeiem comportamento de busca e ingestão de sal, ou seja, do apetite ao sódio. Portanto, sendo o íon sódio essencial à vida, é necessário haver uma atuação integrada do sistema neuroendócrino e da função renal para que a concentração plasmática de sódio seja mantida em níveis normais (145 mM). A seguir, foram descritos os principais mecanismos envolvidos na regulação do apetite ao sódio.

\section{Mecanismos neurais}

Os barorreceptores e os receptores de volume monitoram constantemente o volume sanguíneo, e, desde que reduções ou aumentos na concentração plasmática de sódio são frequentemente acompanhadas por alterações no volume sanguíneo, pode-se dizer que esses receptores também contribuem com o controle do apetite ao sódio. Porém, há algumas divergências na literatura sobre o envolvimento dos barorreceptores no controle da ingestão de sódio. A desnervação sinoaórtica, que elimina o sinal aferente dos barorreceptores para o sistema nervoso central (SNC), reduziu significantemente a ingestão de sódio estimulada por furosemida ${ }^{8}$. No entanto, lesões que acometem a região que recebe as projeções aferentes dos barorreceptores, ou seja, na região dorsolateral dos núcleos do trato solitário (NTS), não geram alterações na ingestão de sódio ${ }^{9}$. Esses resultados mostram que a integridade dos barorreceptores e dos receptores de volume não é crucial para o desencadeamento de comportamentos ingestivos frente a perturbações na volemia, sendo este apenas um dos sinais envolvidos no controle do apetite ao sódio.

Enquanto os barorreceptores monitoram indiretamente a mudanças no volume sanguíneo, os osmorreceptores centrais são responsá- 
veis por monitorar a concentração de sódio no líquido cerebroespinal (LCE). Diversos estudos mostraram o possível envolvimento dos osmorreceptores centrais no controle da ingestão de sódio. Weisinger et al. ${ }^{10,11}$ mostraram que a infusão intracerebroventricular (i.c.v.) de salina hipertônica reduz rapidamente a ingestão de sódio em ovelhas com deficiência de sódio. Porém, quando a osmolaridade do LCE foi aumentada pela infusão i.c.v. de manitol, que é capaz de reduzir a concentração de sódio, os autores observaram um aumento na ingestão de sódio em ovelhas privadas ou repletas de sódio ${ }^{10,11}$. Esses resultados sugerem a presença de sensores capazes de perceber tanto o aumento quanto a diminuição na concentração de sódio no LCE e que participam da regulação do apetite ao sódio.

Os osmorreceptores e os receptores de estiramento localizados no trato gastrointestinal também parecem influenciar o comportamento de ingestão de sódio e sua principal função seria inibitória. Estudo em ratos com fístula gástrica ${ }^{12}$ mostrou que os animais mantidos com a fístula aberta bebem progressivamente grandes volumes de salina hipertônica em comparação aos animais controle. O que sugere que os animais mantidos com fístula aberta ao terem o fluido ingerido drenado através da fístula perdem algum tipo de sinal inibitório relacionado ao término da ingestão. Esse sinal inibitório pode ser tanto o sinal da distensão gástrica quanto o do aumento da osmolaridade no trato gastrointestinal. Estudos mais recentes mostraram que a inibição da ingestão de sódio ocorre por meio da combinação dessas duas variáveis, ou seja, o aumento da osmolaridade e do volume do trato gastrointestinal ${ }^{13}$. Esses estudos também mostraram que há correlação inversa entre a concentração da solução salina ingerida com a velocidade de esvaziamento gástrico e o enchimento intestinal. Foi observado que o consumo de salina hipertônica lentificava o esvaziamento gástrico e, consequentemente reduzia o enchimento do trato gastrointestinal e a inibição da ingestão de sódio ocorria mais precocemente. No entanto, o consumo de soluções isotônicas ou hipotônicas levava ao rápido esvaziamento gástrico e à distensão gastrointestinal e resultando em aumento do volume total consumido. Foi sugerido que os osmorreceptores viscerais seriam os responsáveis por detectarem a concentração da solução que passa pelo lúmen intestinal e/ou no tecido adjacente, regulando assim a velocidade do esvaziamento gástrico. A importância desses sinais pré-absortivos reside na possibilidade de antecipação de mudanças do balanço hidrossalino, pois a inibição da ingestão de sódio ocorre antes mesmo de haver mudanças na osmolaridade plasmática ${ }^{13}$.

A destruição do ramo gástrico do nervo vago pelo tratamento com a neurotoxina capsaicina em ratos tratados com DOCA resultou em maior ingestão diária de salina hipertônica em relação ao grupo controle $^{14}$. Confirmando a importância do nervo vago na modulação da inibição da ingestão de fluidos. As fibras aferentes do nervo vago se projetam das vísceras para o NTS no bulbo, lesões que acometem a área postrema e o NTS adjacente também resultam em aumento na ingestão de salina hipertônica ${ }^{15}$. Recentemente, estudo de nosso laboratório mostrou que lesões agudas e crônicas centradas na porção comissural do NTS promovem aumento na ingestão de salina hipertônica estimulada por depleção de sódio ${ }^{16}$. Todos esses resultados sugerem que a AP (área postrema) e o NTS sejam os principais locais a receberem os sinais inibitórios gastrointestinais precoces (via nervo vago) gerados pela ingestão de fluidos.

As informações gustatórias são conduzidas para o SNC por meio de três principais nervos: o vago (X), o glossofaríngeo (IX) e o facial (VII). A transecção bilateral do nervo da corda timpânica (CT), que é um ramo do nervo facial, reduz significantemente a robusta ingestão de sódio observada em animais depletados deste íon mantidos com fístula gástrica aberta ${ }^{12}$. Esse resultado sugere que o nervo da CT fornece sinal, provavelmente excitatório, para gerar a ingestão de sódio observada em animais depletados que perderam os sinais pós-ingestivos. Ademais, os osmorreceptores centrais são responsáveis por monitorar a concentração de sódio no líquido cerebroespinal (LCE).

Diversas áreas no SNC estão envolvidas na regulação do apetite ao sódio, dentre tantas é possível citar: a área postrema (AP), os núcleos do trato solitário (NTS), o núcleo parabraquial (PBN), o núcleo leito da estria terminal (BNST), a amígdala e estruturas da lâmina terminal: o órgão subfornical (SFO), estruturas localizadas na região anteroventral do terceiro ventrículo (AV3V) (que compreendem: órgão vasculoso da lâmina terminal - OVLT e o núcleo pré-óptico mediano $-\mathrm{MnPO})$, entre outras ${ }^{17}$. A AP é um órgão circunventricular localizado na porção caudal do quarto ventrículo $(4 \mathrm{~V})$, sua principal função na regulação do apetite ao sódio está relacionada à inibição desse comportamento. Lesões centradas na AP de ratos produzem aumento significante na ingestão espontânea de salina hipertônica ${ }^{15}$. No entanto, observouse que lesões de AP que comprometiam parte do NTS imediatamente adjacente resultavam em diferentes respostas de ingestão de sódio, e que esta resposta dependia do tamanho da lesão no $\mathrm{NTS}^{15}$. Animais com lesão de AP que acometia pequena parte dos subnúcleos medial e subpostremal do NTS resultavam em grande ingestão espontânea de salina hipertônica, porém, quando a extensão da lesão era maior ocorria apenas discreto aumento na ingestão espontânea de salina hipertônica ${ }^{15}$. Esses resultados mostraram a participação dessas estruturas no controle da ingestão de sódio, no qual a AP exerce função inibitória, provavelmente modulando a atividade excitatória dos neurônios do NTS sobre o apetite ao sódio.

Recentemente, estudos neuroanatômicos descobriram um grupamento de neurônios sensíveis e seletivos à ação da aldosterona localizados exclusivamente no $\mathrm{NTS}^{18}$. Esses neurônios são as únicas células do SNC que expressam o receptor mineralocorticoide (MR) em colocalização com a enzima $11 \beta$-hidroxiesteroide desidrogenase tipo 2 (HSD2), e devido à essa característica, foram denominados de neurônios HSD2. A função da enzima HSD2 consiste em catalisar a conversão dos glicocorticoides endógenos, cortisol (em humanos) e corticosterona (em roedores) em seus análogos biologicamente inativos 11-ceto (11-cetocortisona e 11-dehidrocorticosterona, respectivamente), evitando a ligação de glicocorticoides no $\mathrm{MR}^{19}$. Após confirmarem que essas células 
são sensíveis à aldosterona, Geerling et al. ${ }^{20}$ também evidenciaram que os neurônios HSD2 estão envolvidos na regulação do apetite ao sódio. Esses neurônios apresentam um padrão de ativação estritamente relacionado à sinalização da deficiência de sódio no organismo ${ }^{19,20}$. Outro dado que reforça o envolvimento dos neurônios HSD2 no controle do apetite ao sódio é o fato de que, após o consumo de salina hipertônica, esses neurônios rapidamente se tornam inativos ${ }^{20}$. Essas descobertas reforçam a participação de áreas bulbares no controle da ingestão de sódio.

O PBN é uma região integrativa localizada na porção dorsolateral da ponte que recebe projeções gustatórias e viscerais do NTS e da AP, retransmitindo essas informações para áreas prosencefálicas relacionadas à ingestão, tais como o BNST, o núcleo central da amígdala (CeA) e o $\mathrm{MnPO}^{17}$. O envolvimento do PBN na regulação do apetite ao sódio parece ter caráter inibitório, pois o antagonismo serotoninérgico nesse núcleo leva ao aumento na ingestão de sódio estimulada por angiotensina II (AII) ou pelo tratamento combinado de furosemida com captopril ${ }^{21}$. No entanto, ao contrário do efeito das lesões de AP e NTS sobre o apetite ao sódio, a ingestão de sódio não é afetada por lesões que acometem o núcleo parabraquial lateral $(\mathrm{LPBN})^{17}$.

O BNST e o CeA são estruturas intimamente interconectadas e conjuntamente com o núcleo medial da amígdala (MeA) recebem o nome de amígdala extendida (ExA). Ambas as estruturas têm função bem estabelecida na modulação da ingestão de sódio. Lesões envolvendo o BNST ou CeA eliminam a ingestão espontânea de sódio e reduzem a ingestão de sódio estimulada pela depleção de sódio ou pelo antagonismo do receptor $\alpha 2$-adrenérgico ${ }^{22}$. Essas áreas se mostram específicas no controle da ingestão de sódio, uma vez que a ingestão de água estimulada por AII ou por injeções subcutânea de salina hipertônica não é afetada pelas lesões em BNST ou CeA ${ }^{22}$. Esses dados sugerem que, além de serem anatomicamente relacionados, o BNST e o CeA também apresentam funções muito semelhantes no controle da ingestão de sódio. Estudos de Sakai et al..$^{23,24}$ reforçaram a função da amígdala no controle da ingestão de sódio. Inicialmente, eles mostraram que a ingestão de sódio estimulada por DOCA, mas não pela adrenalectomia, pode ser inibida pela administração de antisense para MR na amígdala medial ${ }^{23}$. Esses resultados enfatizaram que o envolvimento da amígdala na ingestão de sódio está vinculado aos mineralocorticoides, pois o antisense para MR não reduziu a ingestão induzida por adrenalectomia (situação essa na qual há ausência de produção de mineralocorticoides). Sakai et al..$^{24}$ também mostraram que mineralocorticoides são capazes de estimular rápida ingestão de sódio (após 15 minutos), mas de curto prazo quando injetados na região medial da amígdala. Devido à essa rápida resposta não ser característica dos MRs clássicos que desencadeam ações genômicas, os autores concluíram que essa resposta possivelmente ocorreu devido à ligação do mineralocorticoide em um receptor de membrana, o que desencadeou resposta rápida do tipo não genômica ${ }^{24}$. Porém, até o atual momento, não houve comprovação da existência de um MR de membrana.
A participação de estruturas da lâmina terminal no controle do apetite ao sódio parece não se relacionar com a ação dos mineralocorticoides. Lesões da região AV3V, que incluí o OVLT e o MnPO, inibem a ingestão de sódio induzida por AII, mas ao contrário, aumentam a ingestão de sódio induzida por DOCA nos animais lesionados em relação ao grupo controle ${ }^{25}$. No entanto, há dados conflitantes a respeito da função de áreas prosencefálicas no apetite ao sódio induzido por mineralocorticoides. Alguns estudos mostraram que a infusão de antagonista de MR no sistema ventricular do prosencéfalo foi capaz de reduzir a ingestão de sódio em situações nas quais havia aumento da aldosterona plasmática ${ }^{26-28}$. Já outros estudos não observaram modificações na ingestão de sódio induzida por DOCA em animais que receberam antagonista de MR no ventrículo lateral $(\mathrm{VL})^{22,30}$. Recentemente, estudos de nosso laboratório mostraram que a infusão i.c.v. de aldosterona no VL não modificou a ingestão basal de sódio de ratos ${ }^{31}$, corroborando com esse dado o tratamento com antagonista de MR no mesmo local não afetou a ingestão de sódio induzida pelo tratamento com furosemida ${ }^{32}$.

Importante ressaltar que todas essas áreas se conectam umas com as outras por meio de projeções diretas ou indiretas, como ilustrado pela Figura 1.

\section{Mecanismos hormonais}

Além dos componentes neurais envolvidos na regulação do apetite ao sódio há também os componentes hormonais que estimulam ou inibem esse comportamento. Dentre os principais hormônios relacionados à ingestão de sódio está a AII. Durante os estados de hipovolemia os níveis plasmáticos de AII se elevam rapidamente em paralelo com o aparecimento da sede ${ }^{17}$. Porém, o apetite ao sódio se desenvolve muito mais lentamente após a elevação nos níveis circulantes de $\mathrm{AII}^{17}$. Diversos trabalhos mostraram a ação da AII em áreas prosencefálicas (especialmente em estruturas da lâmina terminal) na estimulação do apetite ao sódio. Experimentos que realizaram a infusão i.c.v. de altas doses de AII (até $6.000 \mathrm{ng} \mathrm{h}^{-1}$ ) observaram impressionante aumento na ingestão de sódio dependente de dose e que persistia com o término da infusão ${ }^{33}$. Avrith e Fitzsimons ${ }^{34}$ realizaram a infusão i.c.v. de baixas doses de AII (1 ou 10 pmol h $\left.{ }^{-1}\right)$ e também observaram aumento na ingestão de sódio, porém, esse aumento praticamente retornou aos valores basais com o término da infusão. Uma injeção de AII (100 ou $1000 \mathrm{pmol}$ ) na área pré-óptica resultou em rápido aumento na ingestão de água, no entanto, a ingestão de sódio se elevou somente após cerca de 18 horas e esse aumento também se relacionou com a dose injetada ${ }^{34}$. Esses estudos também mostraram que o apetite ao sódio induzido pela infusão i.c.v. de AII é específico para o sódio, e não é secundário ao aumento na ingestão de água e nem à natriurese induzida pela $\mathrm{AII}^{33,34}$. No entanto, a AII periférica não é tão importante quanto a central na estimulação do apetite ao sódio, pois a administração periférica de antagonistas do sistema renina- 
angiotensina não alterou a ingestão de sódio de animais depletados ou ADX, já a administração central foi capaz de reduzir ou de abolir o apetite ao sódio estimulado por esses protocolos ${ }^{35,36}$.

Aldosterona é um hormônio esteroide sintetizado a partir do colesterol, produzido e secretado pelas glândulas adrenais por estímulos, como: aumento nos níveis circulantes de AII, aumento na concentração plasmática de potássio e pela ação do hormônio adrenocorticotrófico (ACTH). Os estudos sobre o envolvimento da aldosterona no controle da ingestão de sódio tiveram início com os achados inicias de Curt Richter ${ }^{1}$ em ratos ADX. Posteriormente, Rice e Richter ${ }^{5}$ observaram que ratos intactos apresentavam importante aumento na ingestão de sódio quando tratados com DOCA. Esse resultado foi intrigante, uma vez que animais ADX (sem produção de mineralocorticoides) apresentavam também importante aumento na ingestão de sódio ${ }^{1}$ e quando tratados com DOCA essa ingestão era reduzida a valores basais ${ }^{4}$. A partir de então, diversos estudos se iniciaram a fim de investigar qual o mecanismo que estaria envolvido no apetite ao sódio induzido pelo tratamento com mineralocorticoides.

Passadas algumas décadas, Wolf ${ }^{37}$ confirmou os achados de Richter, nos quais a administração de DOCA em ratos intactos estimulava a ingestão de sódio e em ratos ADX abolia esse comportamento. No entanto, ele observou que a supressão do apetite ao sódio de ratos ADX ocorria com doses baixas $(11 \mathrm{mg} / \mathrm{kg})$ de DOCA, porém quando esses animais recebiam maiores doses $(>100 \mathrm{mg} / \mathrm{kg})$ eles novamente apresentavam aumento na ingestão de sódio ${ }^{37}$; sugerindo que animais ADX ainda se mantinham responsivos ao efeito natrorexigênico da DOCA, no entanto, necessitavam de maiores doses do que os animais intactos.

Em experimentos subsequentes, Wolf e Handa ${ }^{38}$ investigaram se o efeito da aldosterona sobre o apetite ao sódio de ratos seria o mesmo que o visto com o tratamento com DOCA. Os autores utilizaram doses menores de aldosterona (30, 60 e $120 \mu \mathrm{g} /$ semana), uma vez que as doses de DOCA $(2,5 \mathrm{mg} / \mathrm{dia})^{5,37}$ estavam muito acima do valor fisiológico. Os ratos receberam apenas uma injeção das doses supracitadas durante quatro semanas, totalizando quatro injeções. O resultado obtido foi que o tratamento com aldosterona estimulou aumento na ingestão de sódio que mostrou ser dependente de dose ${ }^{38}$. No entanto, os autores deixaram muitas dúvidas em relação a esse resultado. Com o objetivo de evitar a inibição do apetite ao sódio pela retenção de sódio, os ratos foram mantidos em dieta hipossódica durante todo o tratamento com aldosterona. Isso pode ter mascarado o real efeito do tratamento crônico com baixas doses de aldosterona, uma vez que a dieta hipossódica é sabidamente um potente estímulo para o apetite ao sódio ${ }^{2}$. A concentração da solução salina utilizada para o teste do apetite ao sódio é outra questão duvidosa, os autores utilizaram solução isotônica $(0,15$ $\mathrm{M}$ de $\mathrm{NaCl}$ ), que é preferencialmente consumida pelos ratos nos testes de preferência-aversão ${ }^{39}$, e os autores ainda relatam que em estudos preliminares o tratamento com aldosterona não foi capaz de estimular a ingestão de salina mais concentrada ${ }^{38}$. Portanto, esse estudo não mos-

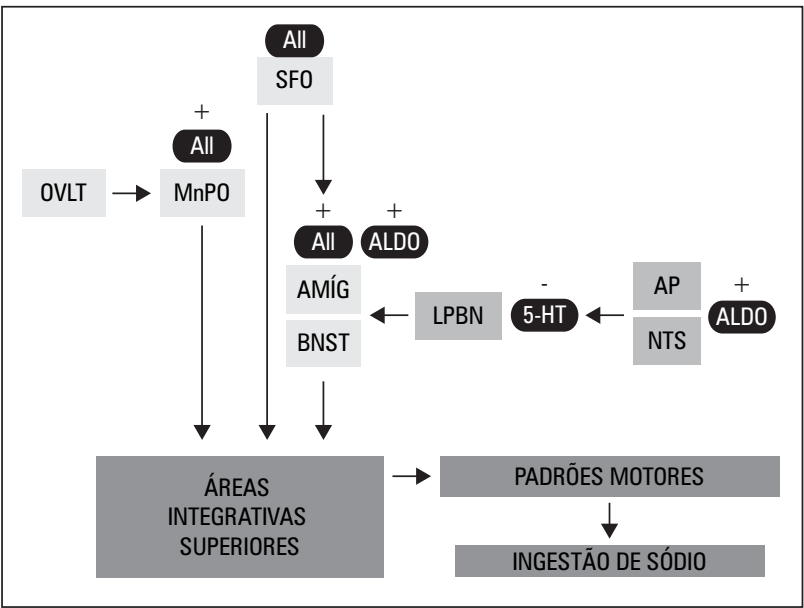

Figura 1: Esquema mostrando as conexões entre as principais áreas envolvidas na regulação neural da ingestão de sódio. Branco: áreas romboencefálicas; preto: áreas prosencefálicas; 5 -HT: serotonina; All: angiotensina II, ALDO: aldosterona; AMÍG: amígdala; AP: área postrema; BNST: núcleo leito da estria terminal; LPBN: núcleo parabraquial lateral; MnPO: núcleo pré-óptico mediano; NTS: núcleos do trato solitário; OVLT: órgão vasculoso da lâmina terminal; SFO: órgão subfornical; +: estimulatório; -: inibitório

trou claramente se a aldosterona em baixas doses é capaz de estimular o apetite ao sódio assim como faz o tratamento com doses suprafisiológicas de DOCA.

Weisinger e Woods ${ }^{40}$ mostraram que a injeção subcutânea de aldosterona $(15,150$ ou $1500 \mu \mathrm{g} / \mathrm{kg})$ foi capaz de aumentar a ingestão de sódio $(0,33 \mathrm{M})$ em ratos, mas que essa resposta foi independente da dose administrada. Porém, o valor da ingestão durante o teste de 20 minutos foi muito baixo, por exemplo, os ratos controle beberam em média $0,8 \pm 0,37$ contra $2,5 \pm 0,36 \mathrm{~mL}$ dos ratos que receberam a maior dose de aldosterona $(1500 \mu \mathrm{g} / \mathrm{kg})^{40}$. Os autores ainda sugerem que o apetite ao sódio estimulado por aldosterona possa ser comportamento condicionado, uma vez que ratos com livre acesso à salina hipertônica não apresentaram apetite ao sódio após receberem a injeção de aldosterona, já os animais sem acesso à salina desenvolveram apetite ao sódio após a injeção de aldosterona ${ }^{40}$. Nesse caso, o que talvez tenha acontecido foi que os animais com livre acesso à salina já estavam adaptados e a injeção de aldosterona não teve efeito sobre a ingestão de sódio, e os ratos que não tinham acesso à salina a consumiram por ser uma solução nova para o seu paladar e não por influência da aldosterona. Todos esses estudos deixaram muitas questões a serem esclarecidas e não conseguiram reproduzir com baixas doses de aldosterona o apetite ao sódio que é estimulado pelo tratamento crônico com doses suprafisiológicas de DOCA. E o mecanismo pelo qual a aldosterona atua no controle do apetite ao sódio não pôde ser esclarecido.

Como descrito no tópico anterior, diversos estudos sugerem áreas do SNC como sendo alvos da ação da aldosterona sobre o apetite ao sódio. No entanto, apenas um estudo avaliou a resposta de ingestão de sódio estimulada pela administração central de aldosterona. Sakai et $a l .^{24}$, por meio de implantes bilaterais de aldosterona na amígdala 
de ratos, observaram que esse tratamento desencadeou resposta de ingestão de sódio. No entanto, a resposta encontrada não conseguiu reproduzir os resultados obtidos com o tratamento periférico com mineralocorticoides. A administração de aldosterona na amígdala desencadeou rápida ingestão de sódio (após cerca de 15 minutos da administração) e de curta duração (cerca de 30 minutos), o que caracteriza resposta não genômica que não é característica do MR nuclear, pois sua ativação normalmente desencadeia respostas genômicas ${ }^{19}$. Além disso, a ingestão de sódio promovida por esse tratamento alcançou valores muito inferiores aos observados com o tratamento periférico (cerca de $2 \mathrm{~mL})^{24}$.

Com a caracterização dos neurônios HSD2 como as únicas células do SNC sensíveis e seletivas à aldosterona ${ }^{18}$ e estritamente relacionadas ao desenvolvimento do apetite ao sódio ${ }^{20}$, optamos por avaliar se a administração de aldosterona em área próxima à restrita localização desses neurônios (NTS medial ao redor da borda do quarto ventrículo $-4 \mathrm{~V}$ ) seria capaz de desencadear ingestão de sódio em ratos. Como resultados, foi observado que infusão de baixas doses de aldosterona (1, 10 e $100 \mathrm{ng} / \mathrm{h}$ ) no $4 \mathrm{~V}$ durante seis dias consecutivos resultou em robusta ingestão de sódio dependente de dose (chegando a $130 \mathrm{~mL} / 24 \mathrm{~h})^{31}$. Para avaliar se essa resposta não foi fruto de difusão da droga para áreas suprabulbares, repetiu-se o protocolo de infusão no VL e observou-se que esse tratamento não alterou a ingestão basal de sódio nos animais ${ }^{31}$. Destacando, portanto, que os efeitos observados pela infusão de aldosterona no $4 \mathrm{~V}$ foram dependentes da ação em áreas próximas ao local de infusão. Corroborando com nossos achados, observou-se também que a microinjeção de antagonista de MR (RU 28318) no 4V, mas não no VL, reduziu significantemente a ingestão de sódio estimulada pelo tratamento prévio com o diurético furosemida. A importância dos MRs localizados em áreas bulbares próximas ao $4 \mathrm{~V}$ também pôde ser comprovada pela redução significante na ingestão de sódio estimulada pela privação de sódio após a microinjeção de RU 28318 no 4V $\mathrm{V}^{32}$. Esses resultados são as primeiras evidências funcionais a mostrarem que aldosterona atua em áreas bulbares próximas ao $4 \mathrm{~V}$ para estimular o apetite ao sódio. Além disso, esse também foi o primeiro resultado a confirmar que a administração central de aldosterona é capaz de estimular a ingestão de sódio, assim como o tratamento periférico. Importante destacar que o tratamento central com baixas doses de aldosterona no $4 \mathrm{~V}$ é capaz de gerar robusta ingestão de sódio comparável (senão superior) à ingestão de sódio desencadeada pelo duplo tratamento AII e aldosterona ${ }^{41}$. Esses resultados sugerem que áreas bulbares (possivelmente os neurônios HSD2) seja o local preferencial de ação da aldosterona no controle do apetite ao sódio.

Como descrito acima, a AII e a aldosterona atuando sozinhas são capazes de estimular o apetite ao sódio ${ }^{5,20,31,33,34,36,37}$. No entanto, o aumento na ingestão de sódio produzido pelo tratamento com ambos os hormônios é de maior magnitude do que quando AII ou DOCA são administradas em maiores doses individualmente ${ }^{41}$. A partir dessas observações, foi elaborada a teoria do sinergismo, que propôs que o apetite ao sódio é o resultado da elevação simultânea nas concentrações de aldosterona periférica e AII produzida centralmente ${ }^{42}$. Essa elevação simultânea de ambos os hormônios ocorre naturalmente, por exemplo, quando o animal é mantido em dieta deficiente de sódio ${ }^{43}$. Como demonstrado inicialmente por Fluharty e Epstein ${ }^{41}$, a infusão i.c.v. de baixas doses de AII associada ao prétratamento periférico com mineralocorticoides (DOCA) estimula o apetite ao sódio, que é superior à soma das ingestões produzidas por doses maiores de cada hormônio administradas individualmente. Corroborando com essa teoria, o apetite ao sódio induzido por furosemida só pôde ser abolido pelo tratamento combinado de um antagonista de MR (i.c.v.) com a administração periférica do inibidor da enzima conversora de angiotensina (captopril) ${ }^{26}$. Sendo que o tratamento isolado com cada uma dessas drogas apenas reduziu a ingestão de sódio ${ }^{26}$. Estudos de nosso laboratório corroboram com esses resultados, pois o apetite ao sódio induzido por furosemida e por dieta hipossódica, situações nas quais há aumento tanto de AII quanto de aldosterona circulantes ${ }^{43,44}$, apenas foi reduzido pelo bloqueio do MR bulbar ${ }^{32}$.

Além da aldosterona e da AII, os glicocorticoides também mostram importante interação com esses hormônios na indução do apetite ao sódio. Os glicocorticoides potenciam o apetite ao sódio estimulado por aldosterona quando administrados simultaneamen$\mathrm{te}^{45}$, inclusive em animais $\mathrm{ADX}^{37}$. No entanto, quando administrados individualmente os glicocorticoides não induzem à ingestão de sódio ${ }^{37,45}$. Ma et al..$^{45}$ sugeriram que os glicocorticoides podem atuar no SNC, aumentando o número de MRs aos quais se ligam à aldosterona e, com isso, potenciam a ingestão de sódio induzida pelos mineralocorticoides.

Assim como a AII e a aldosterona atuam para estimular o apetite ao sódio, alguns outros hormônios atuam no sentido oposto, ou seja, inibindo esse comportamento. A ocitocina (OT) e a vasopressina (AVP) são sintetizadas nos neurônios magnocelulares do núcleo paraventricular (PVN) e do núcleo supraóptico (SON) do hipotálamo e são liberadas para a circulação pela hipófise posterior $^{17}$. A OT e a AVP são secretadas simultaneamente em resposta à hiperosmolaridade e à hipovolemia, e, quando administradas sistemicamente, induzem a natriurese. Evidências sugerem que a OT mantenha o apetite ao sódio inibido em circunstâncias nas quais o consumo de água seja mais urgente, como é o caso da desidratação celular ou na hipovolemia severa ${ }^{17}$. Sendo assim, após o consumo de água, a diluição osmótica no LEC inibe a secreção pituitária de OT, o que culmina com o surgimento do apetite ao sódio. A sugestão inicial para a função inibitória da OT foi a observação de que sua secreção tem relação inversa com o apetite ao sódio ${ }^{46}$. Além disso, administração i.c.v. de OT, mas não a periférica ${ }^{46}$, inibe a ingestão de sódio em ratos hipovolêmicos sem afetar a ingestão de água ${ }^{47}$. O que sugere que a OT central, mas não a periférica, 
seja a responsável por inibir os circuitos cerebrais que induzem o apetite ao sódio.

O hormônio AVP tem como principal função a conservação renal de água por meio de sua ação antidiurética ${ }^{17}$. No entanto, sua função na inibição da ingestão de sódio não é tão bem esclarecida como a da OT. Uma vez que, a injeção i.c.v. de AVP reduziu a ingestão de sódio em ratos depletados, porém, esta redução se relacionou com alterações motoras provocadas pela injeção de $\mathrm{AVP}^{48,49}$. Além disso, a redução na ingestão não foi específica para o sódio, pois também houve redução na ingestão de sacarose ${ }^{49}$. Outro dado curioso foi que a injeção de antagonista do receptor $\mathrm{V}_{1}$ foi capaz de reduzir a ingestão de sódio, mas não de sacarose, nos ratos depletados sem causar alterações motoras ${ }^{49}$, o que sugere que a AVP possa ter alguma função na gênese do apetite ao sódio.
O peptídeo atrial natriurético (ANP) é um hormônio que é secretado na circulação sanguínea durante a expansão de volume e também está envolvido na inibição do apetite ao sódio. A administração i.c.v. de ANP inibe a ingestão de sódio em ratos depletados ${ }^{50}$. Além disso, também apresenta importante ação natriurética, inibe a ingestão de água e reduz a liberação de AVP (portanto também tem ação diurética).

Resumindo, desde os achados iniciais de Curt Richter na década de 1930, muito se descobriu sobre os mecanismos envolvidos na regulação do apetite ao sódio, porém, muitas questões permanecem desconhecidas e necessitam de mais estudos. É certo que o apetite ao sódio é comportamento que requer complexa rede de sinais neurais, hormonais e que diversas regiões do SNC estão envolvidas nessa resposta, e a maneira como todos esses sistemas interagem para o desenvolvimento do apetite ao sódio deve ser foco de futuros estudos.

\section{Referências}

1. Richter CP. Increased salt appetite in adrenalectomized rats. Am J Physiol. 1936;115:155-61.

2. Blair-West JR, Coghlan JP, Denton DA, Nelson JF, Orchard E, Scoggins BA, et al. Physiological, morphological and behavioural adaptation to a sodium deficient environment by wild native Australian and introduced species of animals. Nature. 1968;217:922-8

3. Wilkins L, Richter CP. A great craving for salt by a child with cortico-adrenal insufficiency. J Am Med Assoc. 1940;114:866-8.

4. Richter CP, Eckert JF. Mineral metabolism of adrenalectomized rats studied by the appetite method. Endocrinol. 1938;22:214-24.

5. Rice KK, Richter CP. Increased sodium chloride and water intake of normal rats treated with desoxycorticosterone acetate. Endocrinol. 1943;33: $106-15$.

6. Orent-Keiles E, Robinson A, McCollum EV. The effects of sodium deprivation on the animal organism. Am J Physiol. 1937;119:651-61.

7. Wilkins $L$, Richter CP. A great craving for salt by a child with cortico-adrenal insufficiency. J Am Med Assoc. 1940;114:866-8.

8. Thunhorst RL, Lewis SJ, Jonhson AK. Effects of sinoaortic barorreceptor denervation on depletion-induced salt appetite. Am J Physiol. 1994:267:R1043-R1049.

9. Schreihofer AM, Anderson BK, Schiltz JC, Xu L, Sved AF, Stricker EM. Thris and salt appetite elicited by hypovolemia in rats with chronic lesions of the nucleus of the solitary tract. Am J Physiol. 1999;276:R251-R258.

10. Weisinger RS, Considine P, Denton DA, et al. Rapid effect of change in cerebrospinal fluid sodium concentration on salt appetite. Nature. $1979 ; 280: 490-1$

11. Weisinger RS, Considine P, Denton DA, Leksell L, McKinley MJ, Mouw $\mathrm{DR}$, et al. Role of sodium concentration of the cerebrospinal fluid in the salt appetite of sheep. Am J Physiol Integr Comp Physiol. 1982;242:R51R63.
12. Frankmann SP, Sollars SI, Bernstein IL. Sodium appetite in the sham-drinking rat after chorda tympani nerve transection. Am J Physiol Regul Integr Comp Physiol. 1996;271:R339-R345.

13. Stricker EM, Hoffmann ML. Presystemic signals in the control of thrist, salt appetite, and vasopressin secretion. Physiol Behav. 2007:91:404-12.

14. Curtis KS, Stricker EM. Enhanced fluid intake by rats after capsaicin treatment. Am J Physiol Regul Integr Comp Physiol. 1997; 272: R704-R709.

15. Wang T, Edwards GL. Differential effects of dorsomedial medulla lesion size on ingestive behavior in rats. Am J Physiol Regul Integr Comp Physiol. 1997:273:R1299-R1308.

16. Ogihara CA, Schoorlemmer GHM, Colombari E, et al. Changes in sodium appetite evoked by lesions of the commissural nucleus of the tractus solitarius. Braz J Med Biol Res. 2009:42:561-6.

17. Fitzsimons JT. Angiotensin, thrist, and sodium appetite. Physiol Rev. 1998;78:583-686

18. Geerling JC, Kawata M, Loewy AD. Aldosterone-sensitive neurons in the rat central nervous system. J Comp Neurol. 2006b;494: 515-27.

19. Formenti S, Schoorlemmer GHM, Moreira TS, et al. Mecanismos neurais da aldosterona no controle cardiovascular e do equilíbrio hidroeletrolítico. Arc Bras Ciênc Saúde. 2008:33:54-63.

20. Geerling JC, Engeland WC, Kawata M, et al. Aldosterone target neurons in the nucleus tractus solitarius drive sodium appetite. J Neurosci. 2006a:26:411-7.

21. Menani JV, Thunhorst RL, Jonhson AK. Lateral parabrachial nucleus and serotonergic mechanisms in the control of salt appetite in rats. Am J Physiol Regul Integr Comp Physiol. 1996;270:R162-R168.

22. Zardetto-Smith AM, Beltz TG, Johnson AK. Role of the central nucleus of the amygdala and the bed nucleus of the stria terminalis in experimentallyinduced salt appetite. Behav Brain Res. 1994;645:123-34. 
23. Sakai RR, Ma LY, Zhang DM, et al. Intracerebral administration of mineralocorticoid receptor antisense oligonucleotides attenuate adrenal steroid-induced salt appetite in rats. Neuroendocrinol. 1996;64:425-9.

24. Sakai RR, McEwen BS, Fluharty SJ, et al. The amygdala: site of genomic and nongenomic arousal of aldosterone-induced sodium intake. Kidney Int. 2000;57:1337-45

25. Fitts DA, Tejpkes DS, Bright RO. Salt appetite and lesions of the ventral part of the ventral median preoptic nucleus. Behav Neurosci. 1990;104:81827.

26. Sakai RR, Nicolaidis S, Epstein AN. Salt appetite is supressed by interference with angiotensin $\|$ and aldosterone. Am J Physiol, 1986;251:R762-R768

27. Francis J, Weiss RM, Wei SG, Johnson AK, Beltz TG, Zimmerman K, et al. Central mineralocorticoid receptor blockade improves volume regulation and reduces sympathetic drive in heart failure. Am J Physiol Heart Circ Physiol. 2001;281:H2241-H2251.

28. Sullivan MJ, Hasser EM, Moffitt JA, et al. Rats exhibit aldosteronedependent sodium appetite during $24 \mathrm{~h}$ hindlimb unloading. J Physiol. 2004:557:661-70.

29. Janiak PC, Lewis SJ, Brody MJ. Role of central mineralocorticoid binding sites in development of hypertension. Am J Physiol Regul Integr Comp Physiol. 1990;259:R1025-R1034.

30. Rahmouni K, Sibug RM, Kloet ER, Barthelmebs M, Grima M, Imbs JL, et al. Effects of brain mineralocorticoid receptor blockade on blood pressure and renal functions in DOCA-salt hypertension. Eur $\mathrm{J}$ Phamacol. 2002;436:207-16

31. Formenti S, Schoorlemmer GHM, Menani JV, Colombari E. Functional evidence of hindbrain mechanism for aldosterone-induced sodium appetite. Appetite. 2009;52:832

32. Formenti S, Schoorlemmer GHM, Menani JV, et al. Central mineralocorticoid receptor blockade reduces sodium appetite in rats: new evidence for an old effect. Faseb J. 2010;24.

33. Bryant RW, Epstein AN, Fitzsimons JT, et al. Arousal of a specific and persistent sodium appetite in the rat with continuous intracerebroventricular infusion of angiotensin II. J Physiol. 1980;301:365-82.

34. Avrith DB, Fitzsimons JT. Increased sodium appetite in the rat induced by intracranial administration of components of the renin-angiotensin system. J Physiol. 1980;301:349-64.

35. Sakai RR, Chow SY, Epstein AN. Peripheral angiotensin II is not the cause of sodium appetite in the rat. Appetite. 1990a;15:161-70.
36. Sakai RR, Epstein AN. Dependence of adrenalectomy-induced sodium appetite on the action of angiotensin II in the brain of the rat. Behav Neurosci. 1990b;104:167-76.

37. Wolf $G$. Effect of deoxycorticosterone on sodium appetite of intact and adrenalectomized rats. Am J Physiol. 1965;208:1281-5.

38. Wolf G, Handal PJ. Aldosterone-induced sodium appetite: dose-response and specificity. Endocrinol. 1966;78:1120-4.

39. Rowland NE, Morian KR, Nicholson TM, et al. Preference for $\mathrm{NaCl}$ solutions in sham drinking Sprague-Dawley rats: water deprivation, sodium depletion, and angiotensin II. Physiol Behav. 1994;57:753-7.

40. Weisinger RS, Woods SC. Aldosterone-elicited sodium appetite. Endocrinol. 1971;89:538-44

41. Fluharty SJ, Epstein AN. Sodium appetite elicited by intracerebroventricular infusion of angiotensin II in the rat: II. Synergistic interaction with systemic mineralocorticoids. Behav Neurosci. 1983:97:746-58.

42. Epstein AN. Mineralocorticoids e cerebral angiotensin may act together to produce sodium appetite. Peptides. 1982;3:493-4.

43. Fregly MJ, Rowland NE. Role of renin-angiotensin-aldosterone system in $\mathrm{NaCl}$ appetite of rats. Am J Physiol Regul Integr Comp Physiol. 1985;248:R1-R11.

44. Rowland NE, Morian KR. Roles of aldosterone and angiotensin in maturation of sodium appetite in furosemide-treated rats. Am J Physiol Integr Comp Physiol. 1999;276:R1453-R1470.

45. Ma LY, McEwen BS, Sakai RR, etal. Glucocorticoids facilitate mineralocorticoidinduced sodium intake in the rat. Horm Behav. 1993;27:240-50.

46. Stricker EM, Verbalis JG. Central inhibitory control of sodium appetite in rats: correlation with pituitary oxytocin secretion. Behav Neurosci. 1987;101:560-7.

47. Blackburn RE, Stricker EM, Epstein AN. Central oxytocin mediates inhibition of sodium appetite by naloxone in hypovolemic rats. Neuroendocrinol. 1992:56:255-63.

48. Sato MA, Sugawara AM, Menani VJ, et al. Idazoxan and the effect of intracerebroventricular oxytocin or vasopressin on sodium intake of sodiumdepleted rats. Regul Pept. 1997;69:137-42.

49. Flynn FW, Kirchner TR, Clinton ME. Brain vasopressin and sodium appetite. Am J Physiol Regul Integr Comp Physiol. 2002;282:R1236-R1244.

50. Antunes-Rodrigues J, McCann SM, Samson WK. Central administration of atrial natriuretic factor inhibits saline preference in rats. Endocrinol. 1986;118:1726-8. 